\title{
Review of Energy Detection for Spectrum Sensing in Various Channels and its Performance for Cognitive Radio Applications
}

\author{
${ }^{1}$ R. Suresh Babu and ${ }^{2}$ M. Suganthi \\ ${ }^{1}$ Department of ECE, Kamaraj College of Engineering and Technology, \\ Virudhunagar, Tamilnadu-626001, India \\ ${ }^{2}$ Department of ECE, Thiagarajar College of Engineering, Madurai
}

Received 2012-04-28, Revised 2012-05-27; Accepted 2012-08-04

\begin{abstract}
Spectrum sensing is the basic and important operation in Cognitive Radio (CR) to find the unused spectrum. Energy detector is a popular sensing method because it doesn't require transmitted signal properties, channel information, of even the type of modulation. This study summarizes the performance result of energy detector over Additive White Gaussian Noise (AWGN), Rayleigh fading and Nakagami fading channels. Energy detection with soft decision and hard decision are also studied for different number of cognitive nodes as well as each cognitive node having multiple antennas. The performance of hard decision and soft decision are evaluated by means of complementary Receiver Operating Characteristic (ROC) curves. It clearly shows that the probability of missing detection decreases for increasing the number of antennas in cognitive node and also increasing of cooperated cognitive users.
\end{abstract}

Keywords: Cognitive Radio, Spectrum Sensing, Energy Detection, Diversity Reception, Soft and Hard Decision, Rayleigh Fading, Cognitive Radio (CR), Cognitive Nodes

\section{INTRODUCTION}

The idea of cognitive radio has been first introduced by (Mitola and Maguire, 1999). It is defined as software defined radio which is aware of its environment, learns from and has the ability to change its parameters according to these changes in its environment and the network requirements (Haykin, 2005). The name cognitive radio as we use today refers mostly to spectrum aware communication systems. The need for the cognitive radio emerged from the fact that current frequency allocations (with fixed spectrum assignment policy) show that the radio spectrum is highly occupied, i.e. spectrum is a scarce resource, however, it is highly underutilized (i.e., spectrum is not used effectively).

Cognitive radio systems basically consist of primary (licensed) and secondary(unlicensed-cognitive) users, secondary users continuously check the frequency bands to determine if there is a primary user transmitting, if not, the band is available and the secondary user can start transmitting its own data. These spectrum holes can occur in two ways, in time or in space. When a primary user is not transmitting at a given time, then there's a temporal spectrum hole, if, a primary user is transmitting in a certain portion of the spectrum at a given time but it is too far away from the secondary user so that the secondary user can reuse the frequency, then a spatial spectrum hole exists.

The main functions of a cognitive radio can be addressed as follows (Letaief and Zhang, 2009):

- Spectrum sensing is the process of a cognitive radio sensing the channel and determining if a primary user is present, detecting the spectrum holes

- Spectrum management is selecting the best available channel (for a cognitive user) over the available channels.

- Spectrum sharing is the allocation of available frequencies between the cognitive users

- Spectrum mobility is the case when a secondary user rapidly allocates the channel to the primary user when a primary user wants to retransmit again 
Among these functions, spectrum sensing is the one that has driven most interest.

Spectrum sensing methods for a cognitive radio system can be listed as follows.

\subsection{Matched Filter Detection}

This method incorporates a filter matched to the primary user's signal at the cognitive radio receiver. Obviously, this method is optimal in the sense that it maximizes the SNR, minimizing the decision errors. However, this method is not practical since it requires the cognitive user to know the primary user's signaling type.

\subsection{Energy Detection}

This method uses a squaring device followed by an integrator, the output of which gives the decision variable. This variable is then compared with a threshold and if it is above the threshold, then the result of the detector is that a primary user is present. Energy detection is very practical since it requires no information about the primary user's signal. The drawbacks of this system are it has poor performance in low SNR regimes.

\subsection{Cyclostationary Feature Detection}

Uses the built-in periodic components (features) of the modulated signals (carriers). It takes the Cyclic Autocorrelation Function (CAF) of the signal observed and then obtains the Spectral Correlation Function (SCF) from it(by taking the FT of CAF), than it finds the line components corresponding to these frequencies, if there's a primary user, there's line components at frequencies other than zero, otherwise, only line component is at $\mathrm{f}=0$.

\subsection{Covariance Detection}

This method determines if a primary user is present from the covariance matrix of the received signal, it uses the property that the off diagonal elements of the covariance matrix is non-zero when a primary user is present and zero otherwise.

\subsection{Wavelet Detection}

The spectrum of interest is decomposed as a train of consecutive frequency sub bands, wavelet transform is used to detect the irregularities in these bands (PSD is relatively smooth within the sub bands and possess irregularities at the edges between two neighboring sub bands). Wavelet transform carries information about the locations of these frequencies and the PSD of the sub bands. Vacant frequency bands are obtained by detecting the singularities of the PSD of the signal observed, by taking the wavelet transform of its PSD.

\subsection{Cooperative Sensing}

Cooperative sensing is a method in which multiple cognitive radios collaborate either by sending their decision statistics or the final 1 bit decision to a common node (ex. a base station) and the final decision is done by the base station. This method is more powerful than other methods in a sense that it achieves multiuser diversity and mitigates the Hidden Node Problem, which occurs either when a primary user is shadowed by an obstacle, so that the cognitive user cannot detect it, which results in cognitive user to transmit in the same spectrum band with the primary user, causing high interference to it. Cooperative sensing is usually performed by cognitive users each with an energy detector. Cooperation in Cognitive Radios can occur in two ways L, one is Cooperative Sensing which is as described before and the second one is Cooperative Transmission in which either secondary users transmit other secondary users' data or secondary users transmit primary user's data to help its transmission, this is referred as cognitive relay, then the sensing is either done at a common node (which receives both primary and secondary users' data) or secondary users can perform sensing as well as relaying primary user's data (Simeone et al., 2007).

The rest of the study is organized as follows. In the following section, performance of energy detection over fading channel is analyzed. Next, the result of the single antenna case is used for evaluating the performance of multiple antenna cognitive radios (Pandharipande and Linnartz, 2007) and different combing methods when cognitive users are collaborating by sending their decision to common node (Soft and Hard Decision Combining). Conclusion is given in the last section

\subsection{System Model}

The system model is designed with the following considerations:

- All users (whether primary or secondary) have 2 transmit antennas and a single receive antenna

- Users employ Alamouti space time block codes while transmitting. No CSIT, perfect CSIR

- AWGN channel, Rayleigh block fading channel (channels gains are constant over the block period) and Nakagami fading channel are considered for sensing

- Cognitive Radio based nodes (secondary user) will perform energy detection

To start with, consider a low-pass process and a signal with bandwidth W (energy is negligible outside this band), which has an important feature that resulting from the sampling theory. In order to represent the energy of in finite number of terms over a duration $T$, we need approximately $2 \mathrm{TW}$ sample. The energy detector is a noise pre filter followed by a squaring device and integrator that will give the test statistic. 


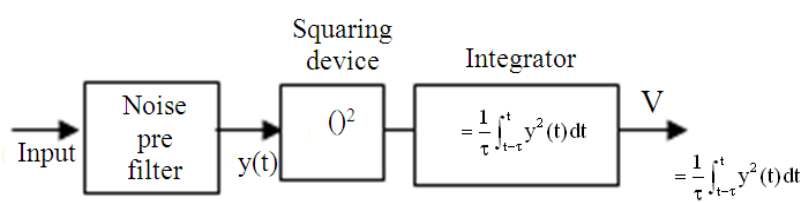

Fig. 1. Energy detector

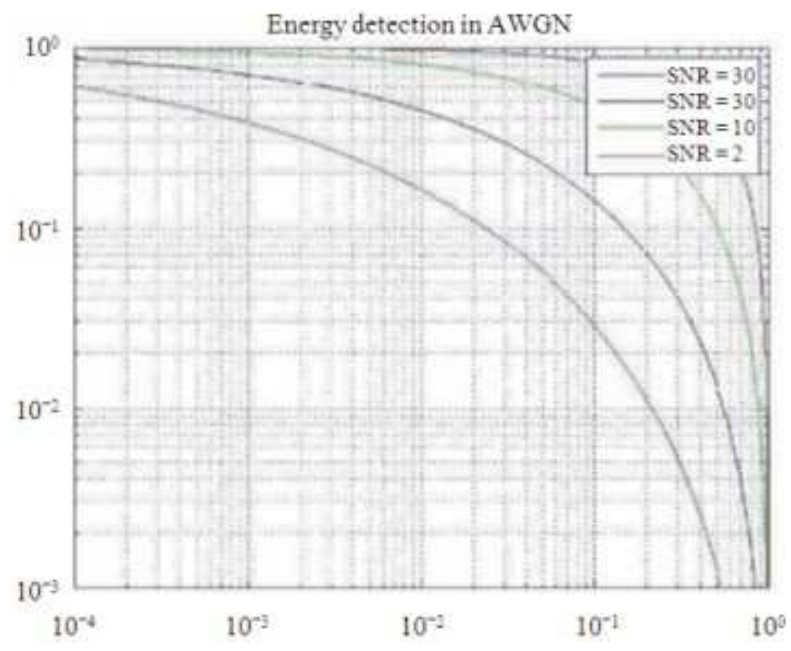

Fig. 2. Energy detection in AWGN channel

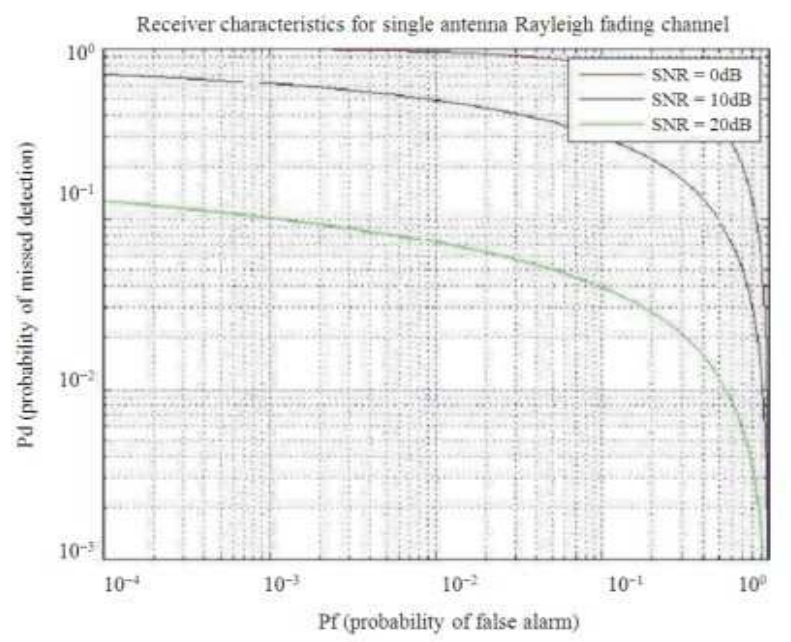

Fig. 3. Energy detection over Rayleigh fading channel

\subsection{Energy Detection Over Awgn Channels}

Energy Detection over AWGN Channels was first studied in (Urkowitz, 1967) in which 2TW samples were used to detect the presence of a signal of duration $\mathrm{T}$ and band limited to $\mathrm{W}$. The basic energy detector is as given in Fig. 1. The decision statistics (output of the detector) is shown to be chi-square distributed with $2 \mathrm{TW}$ degrees of freedom if a signal is not present (primary user not transmitting) and non central chi-square distributed with 2TW degrees of freedom and a noncentrality factor $\mu=$ $\mathrm{E}_{\mathrm{S}} / \mathrm{N}_{\mathrm{o}}$ (SNR-Signal to Noise Ratio) if a user is transmitting.

In this binary hypothesis testing problem, if we let $\mathrm{H} 1$ denote there is a primary user (input is primary user's signal plus noise) and $\mathrm{H} 0$ denote there's no primary user transmitting (input is noise only), the detection (Pd) and false alarm (Pf) probabilities are given as in (1) and (2) where $\lambda$ denotes the threshold and $\gamma$ is defined as $\mu / 2$. The resulting complementary receiver operating characteristic is shown as Fig. 2:

$$
\begin{aligned}
& \operatorname{Pf}=\operatorname{Pr}(\mathrm{v}>\lambda \mid \mathrm{H} \mathrm{l})=\frac{\Gamma(\mathrm{TW} / 2, \lambda / 2)}{\Gamma(\mathrm{TW})} \\
& \operatorname{Pd}=\operatorname{Pr}(\mathrm{v}>\lambda \mid \mathrm{H} 0)=\mathrm{Q}_{\mathrm{TW}}(\sqrt{2 \gamma}, \sqrt{\lambda}) \\
& \operatorname{Pm}=1-\mathrm{Pd}
\end{aligned}
$$

where, Pm denotes the probability of missed detection.

\subsection{Energy Detection Over Rayleigh Fading Channels}

The energy detection over fading channels was studied in (Digham et al., 2003), in which the detection probability for a given SNR $(\gamma)$ was integrated over the pdf of the SNR of the Rayleigh fading channels, which is known to have an exponential distribution. (Note that Pf does not depend on $\gamma$ therefore only Pd needs to be integrated) The closed form of Pd is as given in (3). The performance of the receiver is given in Fig. 3:

$$
\begin{aligned}
\mathrm{f}(\gamma) & =1 / \gamma^{\prime} \exp \left(-\frac{\gamma}{\gamma^{\prime}}\right) \\
\mathrm{Pd} & =\mathrm{e}^{\lambda / 2} \sum_{\mathrm{i}=0}^{\mathrm{N}-2}(\lambda / 2)^{\mathrm{i}} / \mathrm{i} !+\left(1+\gamma / \gamma^{\prime}\right)^{\mathrm{N} / 2-1} *\left[\mathrm{e}^{\lambda / 2(1+\gamma)}\right] \\
& -\mathrm{e}^{\lambda / 2} \sum_{\mathrm{i}=0}^{\mathrm{N}-2}(\lambda / 2)\left(1+\gamma^{\prime}\right) / \mathrm{i} !
\end{aligned}
$$

where, $\gamma^{\prime}$ is the average SNR and N/2 is the time bandwidth product.

\subsection{Energy Detection Over Nakagami Fading Channels}

The energy detection over Nakagami channels is found in by integrating the detection probability for a given SNR over the SNR distribution over Nakagami distribution, the closed form of which is given in (5). The performance over Nakagami fading channels is 
important in a way that when we study the performance of multiple antenna cognitive radios, the performance turns out to be the Nakagami fading performance (with a change of variables), this is because the Nakagami order can be thought as a diversity order. The complementary receiver operating characteristics is as given in Fig. 4. Note that the performance improves as the Nakagami order improves:

$$
\begin{aligned}
& \mathrm{f}(\gamma)=\frac{1}{\Gamma(\mathrm{m})}\left(\frac{\mathrm{m}}{\gamma^{\prime}}\right)^{\mathrm{m}} \gamma^{\mathrm{m}-1} \exp \left(-\frac{\mathrm{m}}{\gamma^{\prime}} \gamma\right), \gamma \geq 0 \\
& \mathrm{Pd}=\mathrm{A}_{1}+\beta^{\mathrm{m}} \mathrm{e}^{-\lambda / 2} * \sum_{1=1}^{\frac{\mathrm{N}}{2}}\left(\frac{\lambda}{2}\right)^{\mathrm{I}} / \mathrm{i} ! \mathrm{F}_{1}\left(\mathrm{~m}, \mathrm{i}+1, \frac{\lambda(1-\beta)}{2}\right)
\end{aligned}
$$

Where:

$$
\beta=\mathrm{m} /\left(\mathrm{m}+\gamma^{i}\right)
$$

$\mathrm{F}_{1}(. ; ;)$ is the confluent hyper geometric function and $\mathrm{m}$ is the Nakagami order:

$$
\begin{aligned}
& \mathrm{A}_{1}=\mathrm{e}^{\lambda \beta / 2 \mathrm{~m}}\left[\beta^{\mathrm{m}-1} \mathrm{~L}_{\mathrm{m}-1}(-\lambda(1-\beta) / 2)+(1-\beta) \sum_{\mathrm{i}=0}^{\mathrm{m}-2} \beta \text { i Li }(-\right. \\
& \lambda(1-\beta) / 2)]
\end{aligned}
$$

\subsection{Energy Detection with Diversity Reception 1.12. Soft Decision Combining 1.13. Square Law Combining}

The energy detection with square law combining is studied in (Digham et al., 2007). In this scheme, the outputs of the square-and-integrate devices are combined which in turn gives a new decision statistic. Under H0 (no primary user), this is a sum of $\mathrm{L}$ central chi-square variables (each having $\mathrm{N}$ degree of freedom) which in turn is another chi-square random variable $\mathrm{LN}$ degrees of freedom. Then the false alarm probability is given as in (6). Under H1 (primary user present), the new decision statistic is a chi-square Random Variable (RV) with LN degree of freedoms, with a non centrality parameter $\gamma_{t}=$ $\Sigma_{\gamma \mathrm{i}}$ the pdf of sum of L i.i.d Rayleigh branches is given as in (4), when every $m$ is replaced by $L$ and each $\gamma^{\prime}$ by $L \gamma$ '. The detection probability is given in (7). This type of combining is defined in (Ghasemi and Sousa, 2007) as Linear Soft Decision Combining. The complementary ROC is given as in Fig. 5:

$\operatorname{Pf}=\frac{\Gamma\left(\frac{\mathrm{LN}}{2}, \frac{\lambda}{2}\right)}{\Gamma(\mathrm{LN} / 2)}$

$$
\operatorname{Pd}=\mathrm{QLN} / 2\left(\sqrt{2 \gamma_{\mathrm{t}}, \sqrt{\lambda}}\right)
$$

\section{L: Number of diversity branches}

\subsection{Square Law Selection}

In Square Law Combining, we select the branch with maximum SNR to make a decision. The detection and false alarm probabilities are given as (8) and (9) (Digham et al., 2007), which are obtained under L independent Rayleigh branches:

$$
\mathrm{Pf}=1-\left[1-\frac{\Gamma\left(\frac{\mathrm{LN}}{2}, \frac{\lambda}{2}\right)}{\Gamma(\mathrm{LN} / 2)}\right]
$$

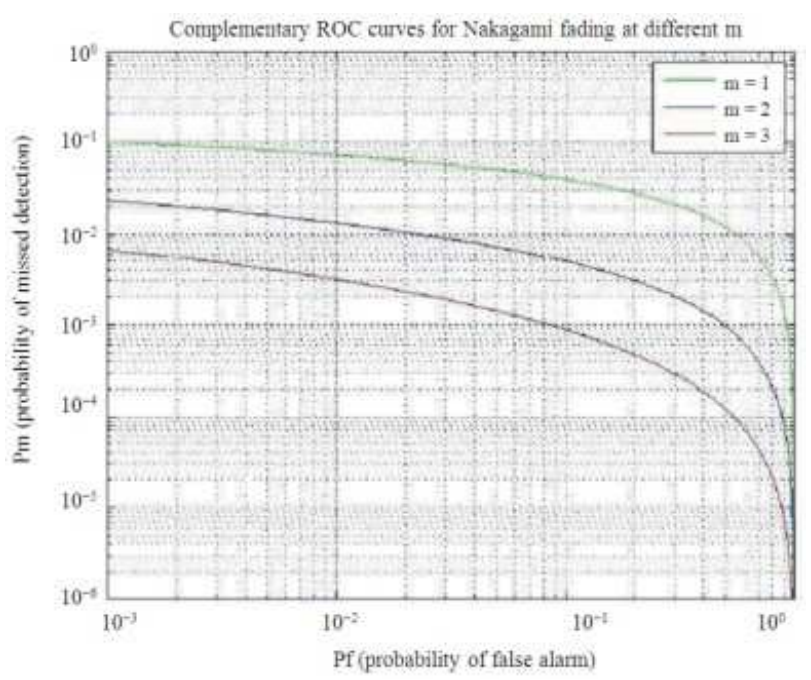

Fig. 4. Energy detection over Nakagami fading channel

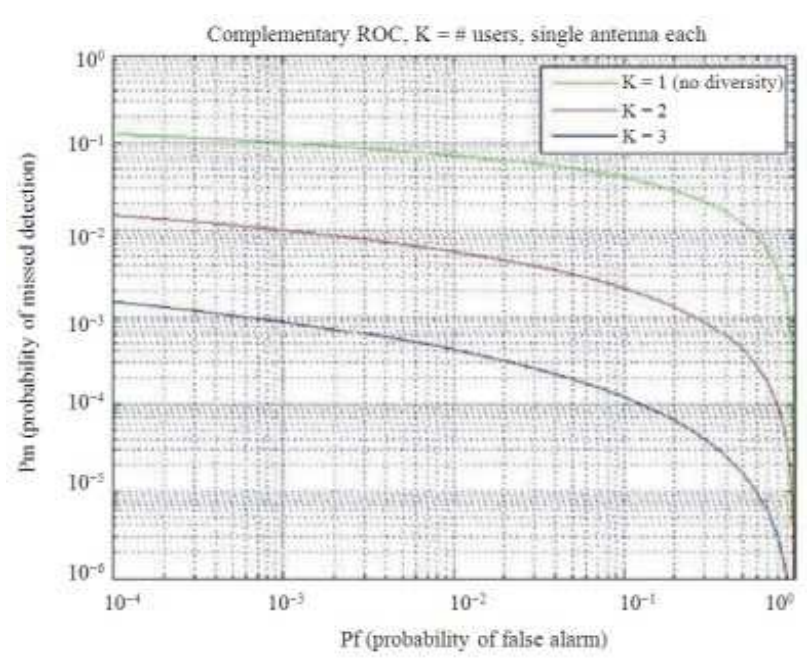

Fig. 5. Complementary ROC for soft decision combining 


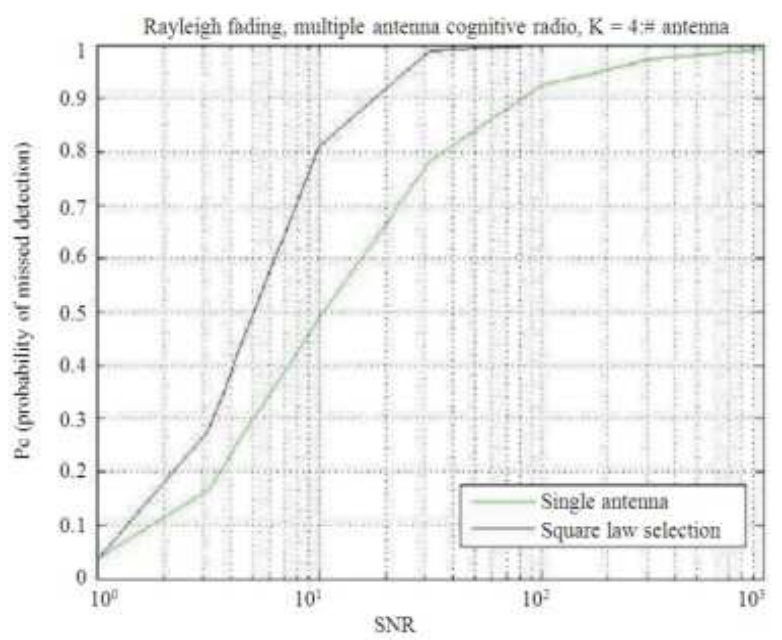

Fig. 6. Multiple antenna cognitive radio

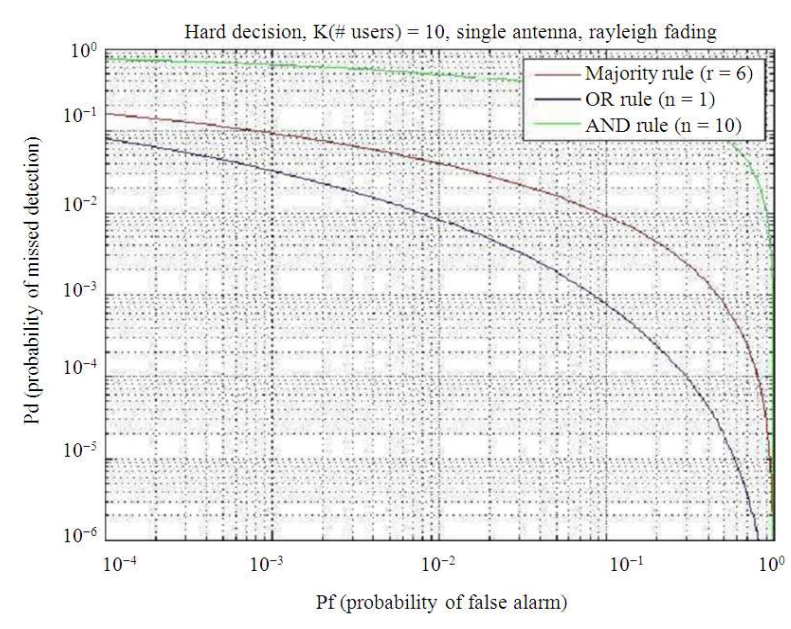

Fig. 7. ROC for Hard decision

$$
\operatorname{Pd}=1-\prod_{i=1}^{\mathrm{L}} \int_{0}^{\infty}\left[1-\mathrm{Q}_{\mathrm{N} / 2}(\sqrt{2} \gamma \mathrm{i}, \sqrt{\lambda})\right] \mathrm{f}\left(\gamma_{\mathrm{i}}\right) \mathrm{d} \gamma_{\mathrm{i}}
$$

Where:

$\mathrm{f}\left(\gamma_{\mathrm{i}}\right)=1 / \gamma_{\mathrm{i}} \exp \left(-\gamma_{\mathrm{i}} / \gamma_{\mathrm{i}}^{\prime}\right), \gamma_{\mathrm{i}} \geq 0$

\subsection{Energy Detection with Multiple Antenna Cognitive Radios}

Performance of multiple antenna cognitive (Pandharipande and Linnartz, 2007) radio depends on the combining type of the branches. While the performance will be as given in Fig. 5 if we use Square Law Combining, if we use Square Law Selection, then the performance is as given in Fig. 6.

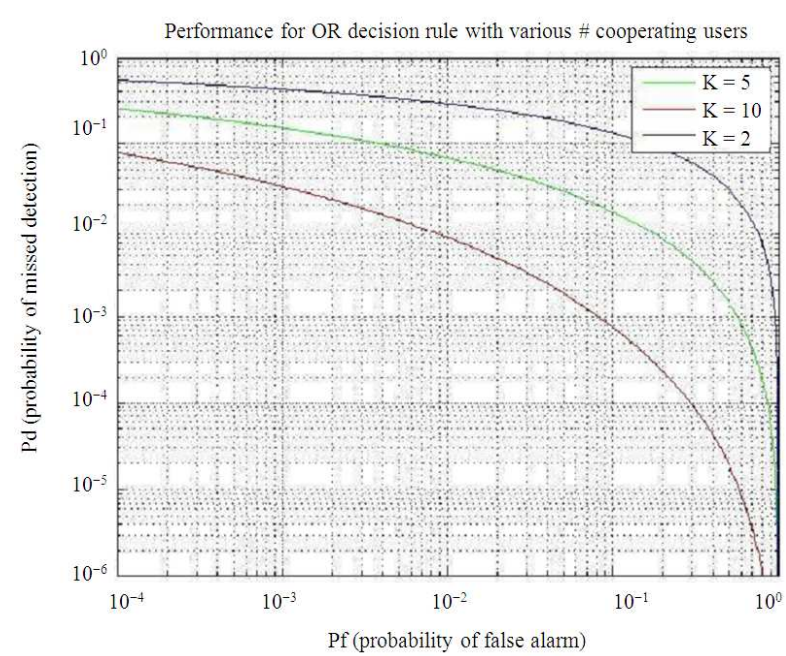

Fig. 8. Performance for OR decision rule

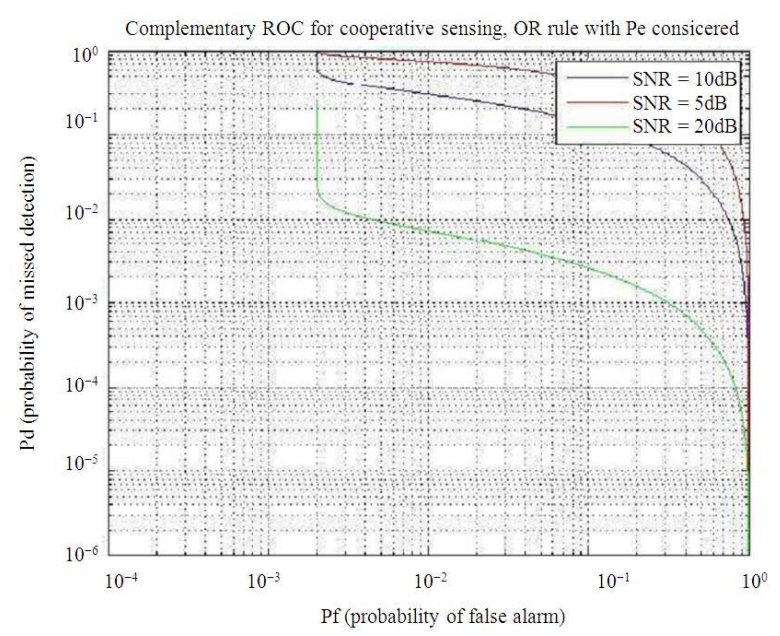

Fig. 9. Performance under Non-ideal reporting channels

\subsection{Hard Decision Combining}

Hard decision is proposed in (Ghasemi and Sousa, 2007) in which different nodes collaborate by sending their final 1-bit decisions to the common node and the common node determines if a primary user is present according to the n-out-of- $\mathrm{K}$ rule, in which the common node decides a primary user is transmitting if $n$ out of $K$ secondary users have supporting decisions. As can be seen in Fig. 7, the OR rule (corresponding to $\mathrm{n}=1$ ) gives the best performance among all combining types.

Although Hard-Decision Combining results in a loss of performance when compared to Soft Decision Combining, hard decision is more practical since it 
requires lower communication overhead (which is important when users collaborate voluntarily rather than enforced) The detection $\left(Q_{d}\right)$ and false alarm $\left(Q_{f}\right)$ probabilities are as given in (10) and (11):

$$
\begin{aligned}
& Q_{d}=\sum_{i=n}^{K} K_{i} P_{d}^{i}(1-P d)^{K-1} \\
& Q_{f}=\sum_{i=n}^{K} K_{i} P_{d}^{i}(1-P f)^{K-1}
\end{aligned}
$$

\section{Where:}

Pd $=$ The detection probability

Pf $=$ The false alarm probability of one node (all nodes are assumed to have the same detection and false alarm probability)

As the number of cooperating users increase, the performance also increases, as given in Fig. 8.

The effect of the non-ideal a channels on the performance of hard-decision with OR rule is as given (Letaief and Zhang, 2009), as in Fig. 9.

\section{CONCLUSION}

Cognitive radio is the promising technique for utilizing the available spectrum optimally. The important aspect of cognitive radio is spectrum sensing and from that identifying the opportunistic spectrum for secondary user communication. In this study, different methods of existing spectrum sensing was studied and the performance of different channels can be presented in terms of Receiver Operating Characteristic (ROC) curves. Finally energy detection based on soft decision and hard decision also presented for different cognitive nodes (secondary users) gives lesser probabilities of missing detection. Further identifying spectrum sensing in angle and code dimensions which gives new research area in cognitive radio applications.

\section{REFERENCES}

Digham, F.F., M. Alouini and M.K. Simon, 2003. On the energy detection of unknown signals over fading channels. Proceeding of the IEEE International Conference on Communication, May 11-15, IEEE Xplore Press, pp: 3575-3579. DOI: 10.1109/ICC.2003.1204119
Digham, F.F., M. Alouini and M.K. Simon, 2007. On the energy detection of unknown signals over fading channels. IEEE J. Trans. Commun., 55: 21-24. DOI: 10.1109/TCOMM.2006.887483

Ghasemi, A. and E.S. Sousa, 2007. Opportunistic spectrum access in fading channels through collaborative sensing. J. Commun., 2: 71-82. DOI: $10.4304 / \mathrm{jcm} .2 .2 .71-82$

Haykin, S., 2005. Cognitive radio: Brain-empowered wireless communications. IEEE J. Selected Areas Commun., 23: 201-220. DOI: 10.1109/JSAC.2004.839380

Letaief, K.B. and W. Zhang, 2009. Cooperative communications for cognitive radio networks. Proc. IEEE, 97: 878-893. DOI: 10.1109/JPROC.2009.2015716

Mitola, J. and G.Q. Maguire, 1999. Cognitive radio: Making software radios more personal. IEEE Personal Commun., 6: 13-18.

Pandharipande, A. and J.P.M.G. Linnartz, 2007. Performance analysis of primary user detection in a multiple antenna cognitive radio. Proceeding of the IEEE International Conference on Communications, Jun. 24-28, IEEE Xplore Press, Glasgow, pp: 64826486. DOI: 10.1109/ICC.2007.1072

Simeone, O., J. Gambini, Y. Bar-Ness and U. Spagnolini, 2007. Cooperation and cognitive radio. Proceedings of the IEEE International Conference on Communications, Jun. 24-28, IEEE Xplore Press, Glasgow, pp: 6511-6515. DOI: 10.1109/ICC.2007.1077

Urkowitz, H., 1967. Energy detection of unknown deterministic signals. IEEE Proc., 55: 523-531. DOI: 10.1109/PROC.1967.5573 